\title{
AdoMet Synergistically Enhances the Chemotherapeutic Action of Cisplatin and Improves Drug Efficacy in JHU-SCC-011 Laryngeal Squamous Cell Carcinoma
}

ISSN: 2637-773X

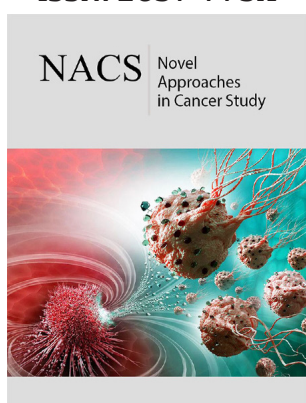

*Corresponding author: Marina Porcelli, Department of Precision Medicine, University of Campania "Luigi Vanvitelli", 80100 Naples, Italy

\section{Submission: 㘹 July 17, 2020}

Published: 淔August 04, 2020

Volume 5 - Issue 1

How to cite this article: Laura Mosca, Martina Pagano, Giovanna Cacciapuoti, Marina Porcelli, et al. Adomet Synergistically Enhances the Chemotherapeutic Action of Cisplatin and Improves Drug Efficacy in Jhu-Scc-011 Laryngeal Squamous Cell Carcinoma. Nov Appro in Can Study. 5(1). NACS.000603. 2020. DOI: 10.31031/NACS.2020.05.000603

Copyright@ Marina Porcelli, This article is distributed under the terms of the Creative Commons Attribution 4.0 International License, which permits unrestricted use and redistribution provided that the original author and source are credited.
Laura Mosca§, Martina Pagano§, Francesca Vitiello, Concetta Paola Ilisso, Alessandra Coppola, Luigi Borzacchiello, Giovanna Cacciapuoti, and Marina Porcelli*

Department of Precision Medicine, University of Campania "Luigi Vanvitelli", 80100 Naples, Italy

\begin{abstract}
Laryngeal squamous cell carcinoma (LSCC), is characterized by an aggressive clinical behavior and lack of specific targeted therapies. Thus, novel therapeutic approaches that take advantage of the combination of anticancer natural compounds and chemotherapeutic molecules currently used for LSCC are strongly necessary. S-adenosyl-L-methionine (AdoMet), the main biological methyl donor, is well known for its antiproliferative, proapoptotic and antimetastatic effect in several type of human cancer. In the current study we investigated the antitumor activity of AdoMet in combination with cisplatin (cDDP), an agent usually used in cancer therapy of LSCC, in JHU-SCC-011 cells and we explored the underlying mechanisms. We found that the combination of $200 \mu \mathrm{M}$ AdoMet/ $0.18 \mu \mathrm{M}$ cDDP synergistically enhanced apoptosis via intrinsic mechanism as confirmed by Western blot analysis of the main proteins involved in this process. Because escape from apoptosis and decreased activation of JNK and ERK are mechanisms of resistance to cDPP, the activation of ERK-1/2 and JNK and the downregulation of the antiapoptotic Bcl-2 protein induced by combined treatments could represent the strategy utilized by AdoMet to potentiate the therapeutic effectiveness of cDDP. The overall data highlighted AdoMet and cisplatin combination as a potential therapy for LSCC in order to improve therapeutic efficacy by reducing side effects and drug resistance mechanisms.
\end{abstract}

Keywords: S-Adenosylmethionine; Laryngeal squamous cancer cells; Head and neck squamous cell carcinoma; Apoptosis; Cisplatin; Drug combination

Abbreviations: AdoMet: S-Adenosyl-L-Methionine; LSCC: Laryngeal Squamous Cell Carcinoma; HNSCC: Head and Neck Squamous Cell Carcinoma; MAPK: Mitogen-Activated Protein Kinase; JNK1: c-Jun N-Terminal Kinase; ERK1/2: Extracellular Signal-Regulated Kinase 1/2; PI: Propidium Iodide; PARP-1: Poly (ADP Ribose) Polymerase, Annexin V-FITC: Annexin V-Fluorescein Isothiocyanate; FACS : FluorescenceActivated Cell Sorting; SD: Standard Deviation; cDDP: Cisplatin

\section{Introduction}

Laryngeal squamous cell carcinoma (LSCC), an epithelial tumor that originates from larynx, is highly aggressive and more than 150.000 new cases are diagnosed each year [1,2]. Alcohol consumption, tobacco use and human papilloma virus (HPV) infections are the main risk factors for these types of cancer $[3,4]$. The main treatment options are surgery, radiotherapy, targeted therapy, and chemotherapy [1,2]. Despite significant progress in finding potential therapeutic targets to improve survival, mortality is still high. Several studies have focused on the discovery of new molecules, obtained from natural compounds, that demonstrate anticancer and anti-proliferation effect and can be used in combination with conventional chemotherapy agents for LSCC treatment. Among natural compounds, our research group focused its interest on S-adenosyl-L-methionine (AdoMet), a naturally occurring sulfur-containing nucleoside found ubiquitously in all living cells where it functions as the main biological methyl donor. The role of AdoMet in various cellular processes, such as proliferation, differentiation, cell cycle regulation, cell migration and apoptosis has been 
amply elucidated [5-9]. In recent years the potential of AdoMet as antiproliferative agent has attracted growing scientific interest in identifying the biological mechanisms and the signal transduction pathways related to the antitumoral activity of this physiological compound alone or in combination with other drugs [10-13]. Nowadays, the major drug used for LSCC treatment is the cisplatin (cDDP), a platinum-based antitumor preparation. The mechanism of action of cDDP is linked to its ability to interact with DNA to form DNA adducts, primarily intrastrand crosslink adducts, which activate several signal transduction pathways and culminate in the activation of apoptosis [14]. The clinical application of this drug is limited because intrinsic or acquired drug resistance during the treatment [15]. Recently our research group have studied the effect of AdoMet/cDDP combination in Cal-33 head and neck squamous cell carcinoma (HNSCC), demonstrating that AdoMet enhances the therapeutic efficacy of cDDP and reduces its resistance mechanisms [16]. We found that the best combination of the two drugs leading to a highly synergistic apoptotic effect at CalcuSyn calculation corresponded to $200 \mu \mathrm{M}$ AdoMet and $0.18 \mu \mathrm{M}$ cDDP after 72 hours of treatment. Noteworthy, the cDDP concentration used in combination with AdoMet, is the lowest effective concentration used so far in anticancer combination treatments of cDDP with natural compounds.

Here, in order to test whether this AdoMet/cDDP combination resulted in a synergistic cytotoxic effect also in LSCC, we evaluated the apoptosis occurrence in JHU-SCC-011 cells demonstrated that the combined treatment with the AdoMet and cDDP sensitizes LSCC cells to the antiproliferative effects of cDDP by enhancing intrinsic apoptotic cell death.

\section{Result and Discussion}

cDDP has in widespread use to treat several forms of cancer, mostly LSCC [17]. In order to improve the pharmacological approaches for the treatment of LSCC we have used AdoMet in combination with cDDP on JHU-SCC-011 cell line and we have analyzed apoptosis occurrence after double labelling with Annexin V-FITC and propidium iodide (PI). Annexin V-FITC was used in conjunction with the vital dye PI to distinguish apoptotic (Annexin V-FITC-positive, PI positive) from necrotic (Annexin V-FITC negative, PI positive) cells [18]. JHU-SCC-011 cells were treated with $200 \mu \mathrm{M}$ AdoMet and $0.18 \mu \mathrm{M}$ cDDP alone or in combination for 72 hours and apoptotic process was evaluated by flow cytometry. As shown in Figure 1, JHU-SCC-011 cells treated with AdoMet showed about $13 \%$ of apoptotic cells, while about $9 \%$ of cell population underwent to apoptosis after 0.18 cisplatin treatment. Notably, the effect of the combined treatment was significantly greater compared to control or to the individual compounds, with apoptosis percentage values of about $31 \%$. The obtained results indicated that, when used together, AdoMet and cDDP were more effective in induction of apoptosis than individual agents confirming that AdoMet was able to synergistically potentiate the cytotoxic effects of cDDP on LSCC cells.

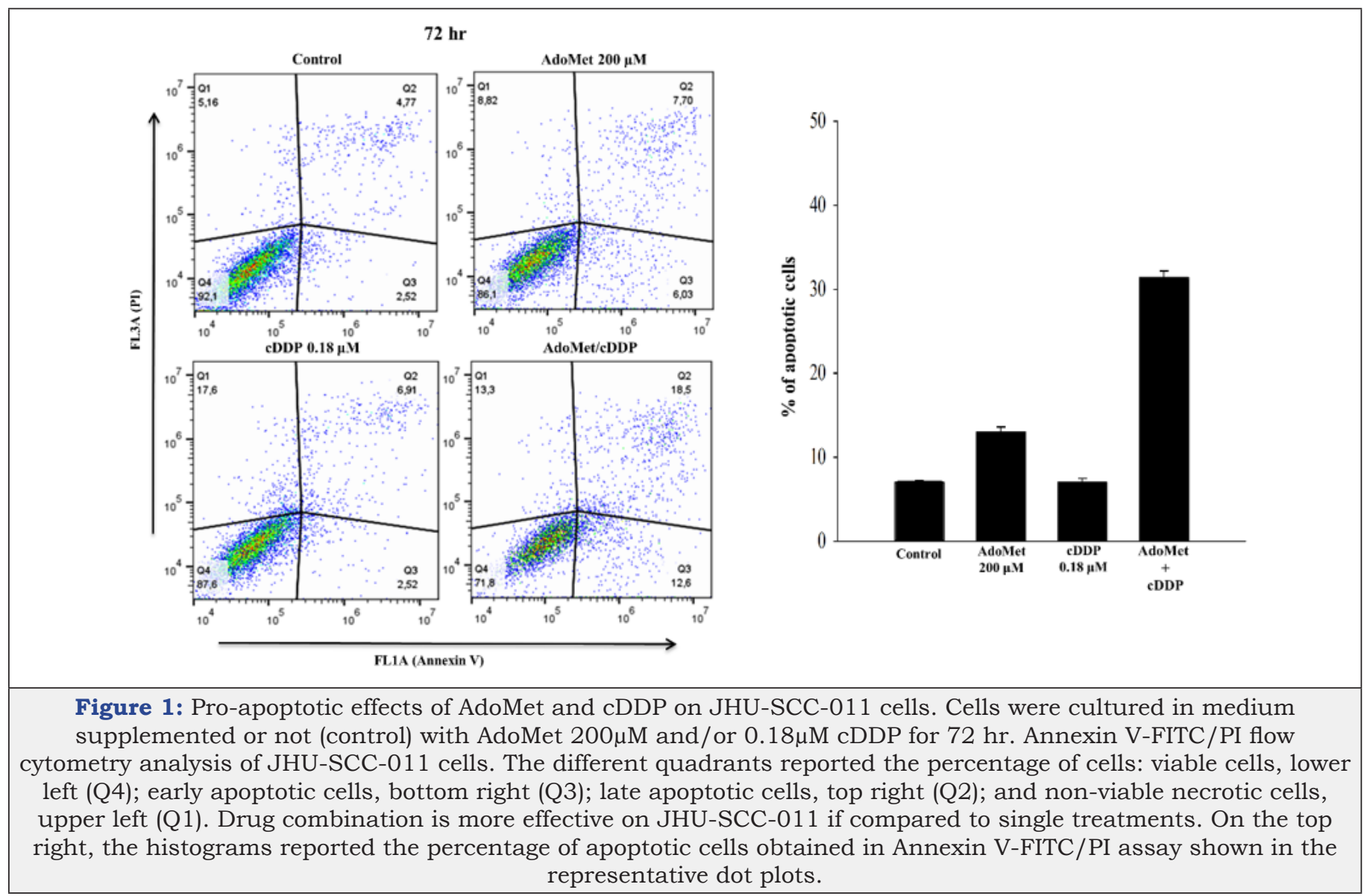


In recent years, several natural compounds have been used in combination with cDDP in order to sensitize cancer cells to platinum compounds and overcome the related resistance mechanisms. Among these, flavonoids based on their ability to affect cancer cells at multiple levels and, thus, to potentially circumvent the development of resistance, emerged as the ideal candidates for cancer chemoprevention. Quercetin, a natural flavonoid found in various plants and foods with well-recognized anti-cancer effect, when used in combination with platinum compound, reduces the effective cDDP concentration to $16.6 \mu \mathrm{M}$ and promotes caspase-dependent extrinsic and intrinsic apoptosis of oral squamous cancer cell lines by inhibiting the activation of NF- $\kappa B$ [19]. Curcumin, the principal compound of turmeric extracted from the plant Curcuma longa, exerts its anticancer effects acting on different pathways involved in the processes of growth, apoptosis and in the different stages of carcinogenesis. This natural compound supports the apoptotic process induced by $10 \mu \mathrm{M}$ of cDDP on two HNSCC lines, through the ERK1/2 and/ or p53 activation [20]. Finally, piperlongumine a natural alkaloid phytochemical and thymoquinone one of the active components of a medicinal plant Nigella sativa were reported as the more efficient molecules used in combination with cDDP in HNSCC until the use of AdoMet [21,22]. In both cases combined treatment experiments reduced the concentration of $\mathrm{cDDP}$ to $5 \mu \mathrm{M}$, however operating with distinct synergistic mechanisms. It has to be noted that the effective cytotoxic cDDP concentration utilized in combination with AdoMet in LSCC is to the best of our knowledge the lowest effective dose never used in combination with natural compounds also in comparison with other types of human cancer [23]. This finding appears particularly interesting since such extremely low sub-toxic concentration of cDDP would result, when in combination with AdoMet, in much greater efficacy than its effective dosage allowing to minimize drug toxicity without affecting its antitumor potency.

In LSCC lines AdoMet in addition to sensitize cells to cDDPinduced apoptosis also synergized with cDDP to inhibit the in vitro migration of Cal-33 and JHU-SCC-011 cells thus providing the basis for including this physiological compound in the therapeutic management of metastatic LSCC [24]. In order to define the mechanisms underlying the apoptotic effect of AdoMet/cDDP combination on JHU-SCC-011 cells, we have analyzed by Western blotting the expression levels of key enzymes involved in the apoptotic cascade, such as caspase-6, caspase-9, PARP-1, Bax, and Bcl-2 proteins and the main MAPK involved in cDDP response including JNK and ERK1/2, at 72 hours from the beginning of the treatment. As shown in Figure 2 AdoMet treatment modified only slightly the levels of apoptotic proteins, while no significant changes were induced by cDDP. On the contrary, the AdoMet/ cDDP combination strongly decreased the levels of uncleaved isoforms of caspase-9, caspase- 6 and their target PARP- 1 with a concomitant increase of Bax/Bcl-2, pJNK/JNK and pERK/ERK1/2 ratio. On the basis of the literature evidence indicating that the activation of MAPK pathways [25,26], and the downregulation of Bcl-2 expression [27] are involved in cDDP-mediated cytotoxicity, our results strongly support the hypothesis that the activation of ERK-1/2 and JNK and the downregulation of Bcl-2 could represent the main strategies utilized by AdoMet to potentiate the therapeutic effectiveness of cDDP. Altogether our results indicate that the combination of AdoMet and cDDP greatly sensitizes JHU-SCC-011 cells to the antiproliferative effects of cDDP by enhancing intrinsic apoptotic cell death.
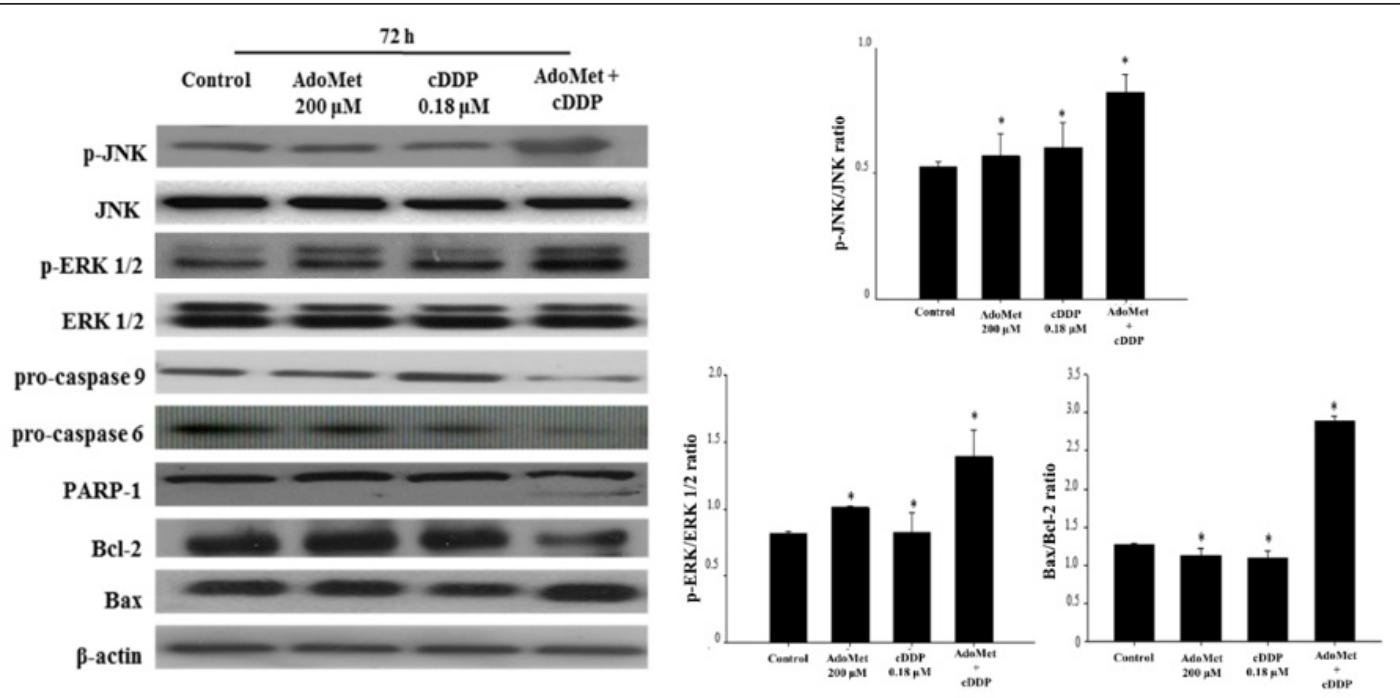

Figure 2: Effects of AdoMet and cDDP on proteins involved in the apoptotic process in JHU-SCC-011 cells. Immunoblot for p-JNK, JNK, p-ERK 1/2, ERK 1/2, pro-caspase 9, pro-caspase 6, PARP-1, Bcl-2 and Bax expression levels, detected by total cell lysates of JHU-SCC-011 cells after 72 hours from the beginning of treatment. The housekeeping protein $\beta$-actin was used as loading control. On the bottom right, the graphs showed the densitometric intensity of p-JNK/JNK, p-ERK/ERK 1/2 and Bax/Bcl-2 bands ratio. The intensities of signals were expressed as arbitrary units. Bars indicated the standard deviations (SDs). ${ }^{*} \mathrm{p}<0.05$ versus control untreated cells. The images are representative of three immunoblotting analyses obtained from at least three independent experiments. 


\section{Conclusion}

The anti-proliferative power of AdoMet has been widely demonstrated in literature, and the current research focuses on the identification of the biological mechanisms and the signal transduction pathways through which the sulfonium compound exerts its chemo-preventive activity. The present mini-review summarizes the recent studies on the anticancer effect of AdoMet in combination with cDDP in LSCC cells and provides information on the mechanisms of action underlying the antitumor effect of this physiological compound that is an approved nutritional supplement and can be used for therapeutic purposes without the common contraindications of chemotherapy drugs. Overall, the data indicated that the combination of AdoMet and cDDP exerted a synergistic proapoptotic effect on LSCC cells and provided further experimental evidence supporting the potential role of this versatile and multifaceted natural compound as an effective adjuvant in conventional cancer chemotherapy.

\section{Acknowledgment}

The work was partially supported by Programme Valere 2020 (VANVITELLI per la Ricerca; Università della Campania "Luigi Vanvitelli").

\section{Competing Interests}

The authors declare no competing interests.

\section{References}

1. Chin D, Boyle GM, Porceddu S, Theile DR, Parsons PG, et al. (2006) Head and neck cancer: Past, present and future. Expert Rev Anticancer Ther 6(7): 1111-1118.

2. Salvador-Coloma C, Cohen E (2016) Multidisciplinary care of laryngeal cancer. J Oncol Pract 12(8): 717-724.

3. Maasland DHE, Vanden Brandt PA, Kremer B, Goldbohm RA, Schouten LJ (2014) Alcohol consumption, cigarette smoking and the risk of subtypes of head-neck cancer: Results from the Netherlands cohort study. BMC Cancer 14: 187.

4. Rampias T, Sasaki C, Psyrri A (2013) Molecular mechanisms of HPV induced carcinogenesis in head and neck. Oral Oncology 50(5): 356-363.

5. Lu SC, Mato JM (2008) S-Adenosylmethionine in cell growth, apoptosis and liver cancer. J Gastroenterol Hepatol 23 (Suppl 1): S73 S77.

6. Martínez N, Varela Rey M, Ariz U, Embade N, Vazquez Chantada M, et al (2008) S-Adenosylmethionine and proliferation: New pathways, new targets. Biochem Soc Trans 36 (Pt 5): 848852.

7. Ilisso CP, Delle Cave D, Mosca L, Pagano M, Coppola A, et al. (2018) S-Adenosylmethionine regulates apoptosis and autophagy in MCF7 breast cancer cells through the modulation of specific microRNAs. Cancer Cell Int 18: 197.

8. Cave DD, Ilisso CP, Mosca L, Pagano M, Martino E, et al. (2017) The anticancer effects of S-Adenosylmethionine on breast cancer cells. JSM Chemistry 5(3): 1049.

9. Ilisso CP, Sapio L, Delle Cave D, Illiano M, Spina A, et al. (2016) S-Adenosylmethionine affects ERK1/2 and Stat3 pathways and induces apoptosis in osteosarcoma cells. J Cell Physiol 231(2): 428-435.

10. Delle Cave D, Desiderio V, Mosca L, Ilisso CP, Mele L, et al. (2018) $\mathrm{S}$-Adenosylmethionine-mediated apoptosis is potentiated by autophagy inhibition induced by chloroquine in human breast cancer cells. J Cell Physiol 233(2): 1370-1383.
11. Ilisso CP, Castellano M, Zappavigna S, Lombardi A, Vitale G, et al. (2015) The methyl donor S-adenosylmethionine potentiates doxorubicin effects on apoptosis of hormone-dependent breast cancer cell lines. Endocrine 50(1): 212-222.

12. Yan L, Tingting B, Linxun L, Quangen G, Genhai S, et al. (2019) S-Adenosylmethionine synergistically enhances the antitumor effect of gemcitabine against pancreatic cancer through JAK2/STAT3 pathway. Naunyn Schmiedebergs Arch Pharmacol 392(5): 615-622.

13. Sun L, Zhang J, Yang Q Si Y, Liu Y, et al. (2017) Synergistic effects of SAM and selenium compounds on proliferation, migration and adhesion of HeLa cells. Anticancer Res 37(8): 4433-4441.

14. Achkar IW, Abdulrahman N, Al-Sulaiti H, Joseph JM, Uddin S, et al. (2018) Cisplatin based therapy: The role of the mitogen activated protein kinase signaling pathway. J Transl Med 16(1): 96.

15. Dasari S, Tchounwou PB (2014) Cisplatin in cancer therapy: Molecular mechanisms of action. Eur J Pharmacol 740: 364-378.

16. Mosca L, Pagano M, Ilisso CP, Cave DD, Desiderio V, et al. (2019) AdoMet triggers apoptosis in head and neck squamous cancer by inducing ER stress and potentiates cell sensitivity to cisplatin. J Cell Physiol 234(8): 1327713291.

17. Le X, Hanna EY (2018) Optimal regimen of cisplatin in squamous cell carcinoma of head and neck yet to be determined. Ann Transl Med 6(11): 229.

18. Vermes I, Haanen C, Steffens Nakken H, Reutelingsperger C (1995) A novel assay for apoptosis. Flow cytometric detection of phosphatidylserine expression on early apoptotic cells using fluorescein labelled Annexin V. J Immunol Methods 184(1): 39-51.

19. Li X, Guo S, Xiong XK, Peng BY, Huang JM, et al. (2019) Combination of quercetin and cisplatin enhances apoptosis in OSCC cells by downregulating xIAP through the NF- $\mathrm{BB}$ pathway. J Cancer 10(19): 4509-4521.

20. Bostan M, Petrică Matei GG, Ion G, Radu N, Mihăilă M, et al. (2019) Cisplatin effect on head and neck squamous cell carcinoma cells is modulated by ERK1/2 protein kinases. Exp and Ther Med 18(6): 50415051.

21. Roh JL, Kim EH, Park JY, Kim JW, Kwon M, et al. (2014) Piperlongumine selectively kills cancer cells and increases cisplatin antitumor activity in head and neck cancer. Oncotarget 5(19): 9227-9238.

22. Alaufi OM, Noorwali A, Zahran F, Al-Abd AM, Al-Attas S (2017) Cytotoxicity of thymoquinone alone or in combination with cisplatin (CDDP) against oral squamous cell carcinoma in vitro. Scient Rep 7: 13131.

23. Baharuddin P, Satar N, Fakiruddin KS, Zakaria N, Lim MN, et al. (2016) Curcumin improves the efficacy of cisplatin by targeting cancer stemlike cells through p21 and cyclinD1-mediated tumor cell inhibition in non-small cell lung cancer cell lines. Oncol Rep 35(1): 13-25.

24. Mosca L, Minopoli M, Pagano M, Vitiello F, Carriero MV, et al. (2020) Effects of S adenosyl L methionine on the invasion and migration of head and neck squamous cancer cells and analysis of the underlying mechanisms. Int J Oncol 56(5): 1212-1224.

25. Sanchez-Perez I, Murguia JR, Perona R (1998) Cisplatin induces a persistent activation of JNK that is related to cell death. Oncogene 16(4): 533-540.

26. Wang X, Martindale JL, Holbrook NJ (2000) Requirement for ERK activation in cisplatin-induced apoptosis. The J Biol Chem 275(50): 39435-39443.

27. Yang D, Zhan M, Chen T, Chen W, Zhang Y, et al. (2017) miR-125b-5p enhances chemotherapy sensitivity to cisplatin by downregulating Bcl2 in gallbladder cancer. Scient Rep 7: 43109. 